\title{
Dupuytren's Contracture With Rare Bilateral Thumb and Little Finger Involvement Demonstrated by Ultrasound Elastography
}

\author{
Aslıhan ULUSOY, ${ }^{1}$ Canan TIKIZ, ${ }^{1}$ Şebnem ÖRGÜÇ² \\ ${ }^{1}$ Department of Physical Medicine and Rehabilitation, Medical Faculty of Celal Bayar University, Manisa, Turkey \\ ${ }^{2}$ Department of Radiology, Medical Faculty of Celal Bayar University, Manisa, Turkey
}

\begin{abstract}
Dupuytren's contracture is a disease that affects the functionality of the hand and causes contractures leading to progressive fibrosis of the palmar fascia. Dupuytren's contracture usually affects the fourth and fifth digits, but thumb involvement is rare. In this article, we presented a rare case of Dupuytren's contracture with bilateral thumb and fifth digit involvement in a 77-year-old male patient who was assessed by ultrasound elastography and gained range of motion after rehabilitation.

Keywords: Dupuytren's contracture; treatment; ultrasound elastography.
\end{abstract}

Dupuytren's contracture (DC) is a benign fibroproliferative disease that affects the palmar fascia. As a result of progressive palmar fascial fibrosis, thickened nodules develop, nodules progress and increase in size to form cords that result in flexion contractures. ${ }^{1,2}$ Restriction of daily activities and hand function impairment may occur depending on the severity and extent of the disease. ${ }^{3}$ In this article, we presented a rare case of DC of bilateral thumb and fifth digit involvement in a male patient who was assessed by ultrasound elastography and had improved range of motion after rehabilitation.

\section{CASE REPORT}

A 77-year-old male patient with a history of posttraumatic fourth finger middle phalanx subtotal amputation (immediate replantation) three months ago was admitted with a complaint of limitation in ring finger range of motion. Besides traumatic finger, physical examination revealed thickened nodules and cord formations overlying the flexor tendons of the first and fifth digits on the palm of both hands that had been causing motion limitation for approximately 10 years and had progressed over time (Figure 1).

Active range of motion was measured with a finger goniometer which provided the following degrees of extension/flexion. Left hand: thumb metacarpophalangeal (MCP) joint -10/40, interphalangeal joint (IPJ) $0 / 40$ and little digit MCP joint 0/80, proximal interphalangeal (PIP) joint $0 / 80$. Right hand: thumb MCP joint $0 / 80$, IPJ $0 / 80$ and little digit MCP joint -20/40, PIP joint $0 / 40$ (a negative value indicating lack of extension at MCP, PIP, and IPJ joints). The Hueston tabletop test was bilaterally positive (if the hand cannot be placed completely flat on the table with the palmar surface, the test is considered positive) and patient was diagnosed with DC. Patient's medical history included high blood pressure, type 2 diabetes mellitus, and coronary artery disease. He had a five pack-year smoking 
history. Hematological examination and blood biochemistry results were normal. Ultrasonography and color Doppler imaging revealed thickening of both tendon sheaths and superficial palmar fascia with high vascularity and heterogeneity. There was no fluid in the tendon sheaths, which is a sign of tenosynovitis. The soft tissue thickening was also more superficial to the tendons located in the palmar fascia and deep surface of the dermis. The increased vascularity was interpreted as representing early signs of nodules and fibrosis which appear during the course of the disease. Nodular soft tissue thickening and thickened aponeurosis coded red on the color scale were harder when compared to the surrounding tissue, which supports fibrosis revealed in sonoelastographic examination (Figure 2, 3). We consulted plastic surgery but surgery was deemed to be appropriate for DC (thumb and little digit) after rehabilitation for post-traumatic ring finger. He was treated for bilateral hands with physical treatment program including contrast bath, ultrasound therapy, palmar massage, range of motion and stretching exercises for 15 days. Patient's complaints decreased significantly. Also, improved hand functionality and increased range of motion were detected after treatment with the following degrees of extension/flexion. Left hand: thumb MCP joint 50, IPJ 20, little digit PIP joint 10 . Right hand: little digit MCP joint 20 , PIP joint 20.

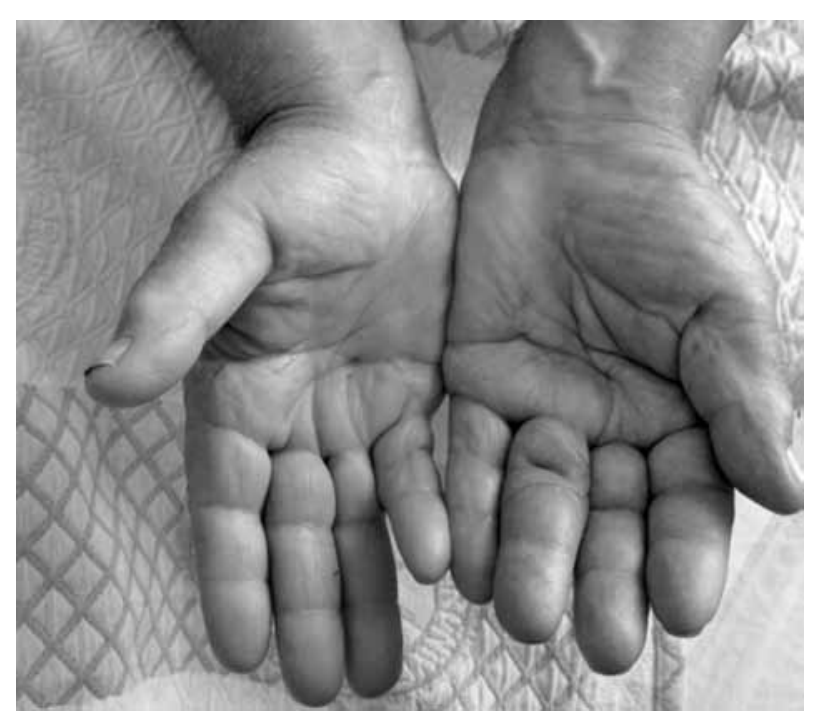

Figure 1. Palmar surface of hand of a 77-year-old male patient with Dupuytren's contracture with bilateral thumb and little finger involvement.

\section{DISCUSSION}

Dupuytren's contracture is more common in middle aged and elderly males with 42 to $60 \%$ of bilateral hand involvement. ${ }^{4}$ It usually affects the ring or little fingers, less commonly the other two fingers, and rarely the thumb. ${ }^{5,6}$ Patients with thumb involvement typically have longer duration time of the disease, are advanced in age, and more likely to have undergone prior operations. ${ }^{6}$ Bilateral involvement and recurrence risks are also found significantly more frequent in patients affected on the radial part of the hand. ${ }^{7}$ Combined involvement of a wide variety of different fingers may be seen in the disease but particularly both thumb and little finger involvement makes the disease challenging in terms of surgical technique and may lead to more restricted hand functionality. ${ }^{8}$

Dupuytren's contracture is usually diagnosed clinically. Magnetic resonance imaging or ultrasound may be useful for the differential diagnosis including tenosynovitis, trigger finger, ganglion cyst, soft tissue mass or for imaging of vascular complications. ${ }^{9}$ Main findings are fibrous nodules and cord-like thickening in palmar fascia. The normal superficial palmar fascia appears as a thin echogenic line overlying the flexor tendons. At ultrasonographic evaluation, an elongated hypoechoic thickened plaque typically continuing with one or more of the flexor tendons and deep surface of the dermis is the pathognomonic sign of the disease. Early nodules are hypoechoic and typically hypervascular in Doppler imaging whereas older nodules are hyperechoic and nonhypervascular due to fibrosis and scar tissue. ${ }^{9}$ Compression sonoelastography which is a newly developed technique as an adjunct to ultrasonographic examination can be used in several musculoskeletal disorders to evaluate the elasticity of muscle structures, tendons, ligaments or soft tissues. ${ }^{10}$ However, the clinical utility still remains under investigation. Elasticity is represented by color coding where areas of stiff tissue appear red and soft tissues appear blue.

Elastography provides information regarding fibrosis. We showed that thickened aponeurosis and nodules had a firm structure with a predominant red color compared to the surrounding tissue in sonoelastographic findings of DC. Sonoelastography is a potential diagnostic 

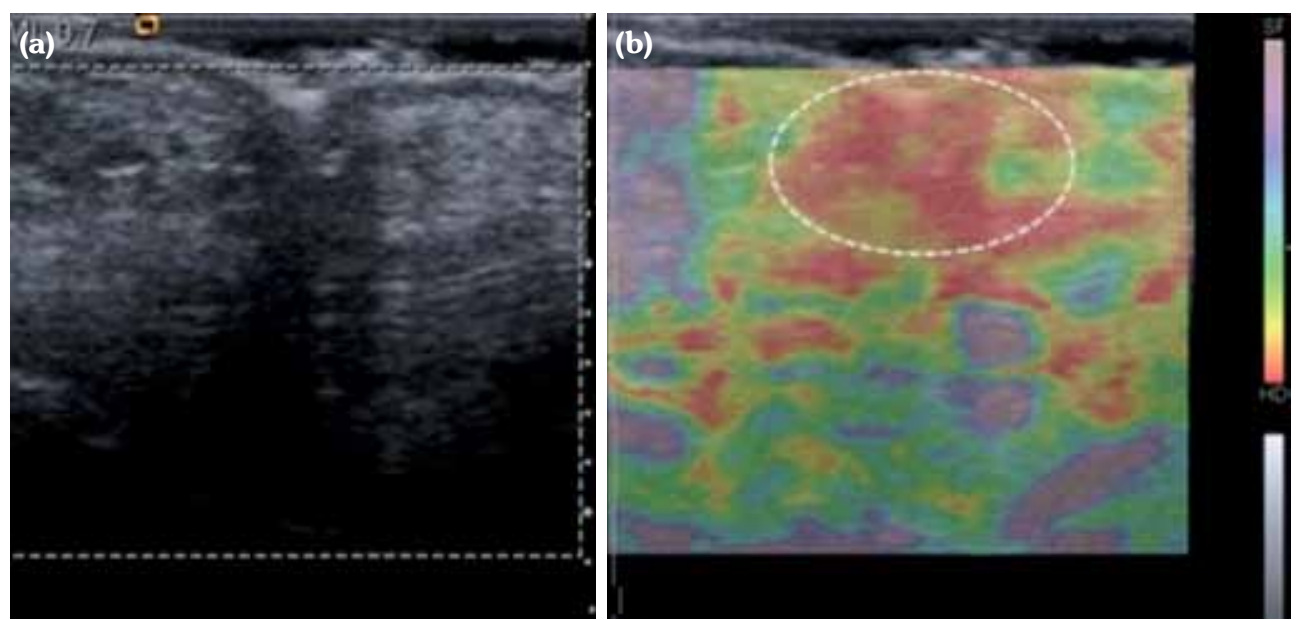

Figure 2. (a) B-mode ultrasonography: Hypoechoic lesions represent nodular soft tissue thickening that causes shrinkage on palmar surface of left hand. (b) Ultrasound elastography: This area coded in red (encircled) is correlated with stiffness of tissue in elastographic evaluation. Color bar indicates relative stiffness of tissues; stiff tissues appear red and soft tissues appear blue.

tool which may differentiate both the acute and chronic findings in DC and provide information regarding differential diagnosis.

The target of the treatment is to improve the functionality of the hand by determining the treatment type. Non-surgical treatment options for DC have been evaluated in clinical trials including intralesional injection of steroid, clostridial collagenase injection (approved by the Food and Drug Administration in 2010 for the treatment of DC), radiotherapy, and interferon gamma injection. ${ }^{1}$ The efficiency of splinting, ultrasound therapy, laser treatments, stretching exercises, and massage treatments have been supported by no more than case reports in the treatment or prevention of recurrence of DC. ${ }^{11,12}$ Generally, surgery for DC should be performed on an affected MCP joint if the contracture is $30^{\circ}$ or greater, or on PIP joint contractures with functional disorder or neurovascular involvement.,13 Our case was appropriate for surgery by contracture of the MCP over $30^{\circ}$, PIP involvement and functional
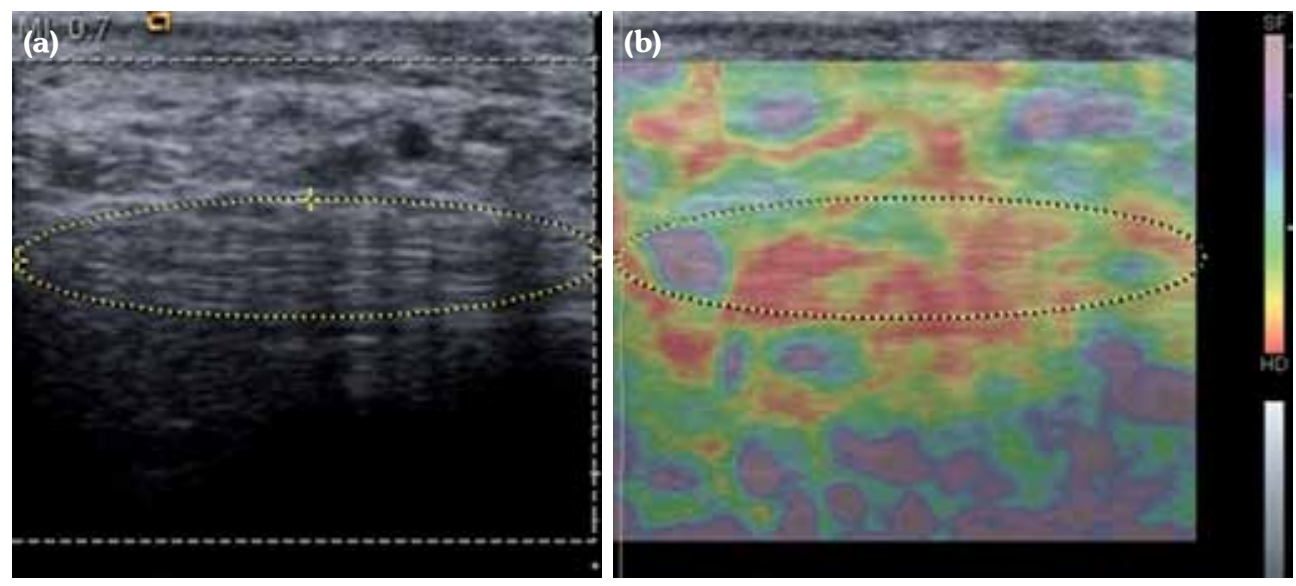

Figure 3. (a) B-mode ultrasonography: Cord-like thickening of left hand palmar fascia and heterogeneous reduced echogenicity. (b) Ultrasound elastography demonstrated that thickened aponeurosis coded in red (encircled) on color scale was harder when compared to surrounding tissue. 
disability. However, surgery was delayed due to the rehabilitation requirement for simultaneous post-traumatic finger injury. Therefore, we included both hands in the physical therapy and therapeutic exercise programs with the goal of providing preoperative contributions for DC. In short term, increased ROM and functionality with this noninvasive treatment may offer an alternative before surgery. On the other hand, this dramatical improvement with physical therapy may be due to the coexistence of diabetic cheiroarthropathy which is characterized by hand stiffness and flexion contractures of the fingers. Tendon sheath thickening may be detected at ultrasonographic evaluation and the Hueston tabletop test may be positive also in both diseases. ${ }^{14}$ Diabetic cheiroarthropathy, that may develop due to the changes in collagen biochemistry and diabetic microangiopathy of the skin, tends to respond well to physical therapy.

Sonoelastography provides a qualitative measurement of stiffness; therefore, we did not base our decision on the type of treatment according to sonoelastographic findings. However, sonoelastography may provide information about the mechanical properties of palmar fascia in addition to morphology and stage of involvement and it should be performed in conjunction with ultrasound examination for the diagnosis of DC. Unfortunately, we were unable to do elastographic assessment to follow-up our patient after treatment. Indeed, elastography may take a role in assessing the efficacy for the treatment of DC objectively by comparing the stiffness values. Further research is needed to support the outcomes of this study.

\section{Declaration of conflicting interests}

The authors declared no conflicts of interest with respect to the authorship and/or publication of this article.

\section{Funding}

The authors received no financial support for the research and/or authorship of this article.

\section{REFERENCES}

1. Shih B, Bayat A. Scientific understanding and clinical management of Dupuytren disease. Nat Rev Rheumatol 2010;6:715-26.

2. Sampson S, Meng M, Schulte A, Trainor D, Montenegro R, Aufiero D. Management of Dupuytren contracture with ultrasound-guided lidocaine injection and needle aponeurotomy coupled with osteopathic manipulative treatment. J Am Osteopath Assoc 2011;111:113-6.

3. Ball C, Pratt AL, Nanchahal J. Optimal functional outcome measures for assessing treatment for Dupuytren's disease: a systematic review and recommendations for future practice. BMC Musculoskelet Disord 2013;14:131.

4. Onat ŞS, Delialioğlu SÜ. Olgu sunumları eşliğinde palmar fibromatozis değerlendirimi ve tedavisi. Turk Osteoporoz Derg 2013;19:52-5.

5. Ellis H. Baron Guillaume Dupuytren: Dupuytren's contracture. J Perioper Pract 2013;23:119-20.

6. Miranda BH, Elliott C, Fahmy FS. Bilateral Dupuytren's contractures of the thumb interphalangeal joints. J Plast Reconstr Aesthet Surg 2012;65:1738-40.

7. Abe Y, Rokkaku T, Ofuchi S, Tokunaga S, Takahashi $\mathrm{K}$, Moriya H. Dupuytren's disease on the radial aspect of the hand: report on 135 hands in Japanese patients. J Hand Surg Br 2004;29:359-62.

8. Flatt $\mathrm{AE}$. The Vikings and Baron Dupuytren's disease. Proc (Bayl Univ Med Cent) 2001;14:378-84.

9. Créteur V, Madani A, Gosset N. Ultrasound imaging of Dupuytren's contracture. J Radiol. 2010;91:68791. [Abstract]

10. Botar Jid C, Vasilescu D, Damian L, Dumitriu D, Ciurea A, Dudea SM. Musculoskeletal sonoelastography. Pictorial essay. Med Ultrason 2012;14:239-45.

11. Degreef I, De Smet L. Dupuytren disease: on our way to a cure? Acta Orthop Belg 2013;79:243-9.

12. Christie WS, Puhl AA, Lucaciu OC. Crossfrictional therapy and stretching for the treatment of palmar adhesions due to Dupuytren's contracture: a prospective case study. Man Ther 2012;17:479-82.

13. Mafi R, Hindocha S, Khan W. Recent Surgical and Medical Advances in the Treatment of Dupuytren's Disease - A Systematic Review of the Literature. Open Orthop J 2012;6:77-82.

14. Cherqaoui R, McKenzie S, Nunlee-Bland G. Diabetic cheiroarthropathy: a case report and review of the literature. Case Rep Endocrinol 2013;2013:257028. 